\title{
Local tirofiban infusion for remnant stenosis in large vessel occlusion: tirofiban ASSIST study
}

Yong-Won Kim', Sung-II Sohn², Joonsang Yoo 2,3, Jeong-Ho Hong ${ }^{2}$, Chang-Hyun Kim', Dong-Hun Kang ${ }^{5}$, Yong-Sun Kim ${ }^{6}$, Seong-Joon Lee ${ }^{7}$, Ji Man Hong ${ }^{7}$, Jin Wook Choi ${ }^{8}$, Yang-Ha Hwang ${ }^{1 *}$ and Jin Soo Lee ${ }^{7^{*}}$ (D)

\begin{abstract}
Background: Compared with embolic occlusions, intracranial atherosclerotic stenosis (ICAS)-related large vessel occlusions (LVOs) often require rescue treatment following mechanical thrombectomy (MT). Herein, we hypothesized that local tirofiban infusion can be effective and safe for remnant stenosis in LVO during endovascular treatment and can improve clinical outcomes.

Methods: This observational multicenter registry study (January 2011 to February 2016) included patients with ICAS who underwent endovascular treatment for LVO within $24 \mathrm{~h}$ after stroke onset. An underlying fixed focal stenosis at the occlusion site observed on cerebral angiography during and after MT was retrospectively determined as a surrogate marker of ICAS. Procedural and clinical outcomes were compared between the tirofiban and nontirofiban groups.

Results: Of 118 patients, 59 received local tirofiban infusion. Compared to the non-tirofiban group, patients were older (non-tirofiban group versus tirofiban group; median, 63 years vs. 71 years, $p=0.015$ ) and the onset-to-puncture time was longer (median, $275 \mathrm{~min}$ vs. $395 \mathrm{~min}, p=0.036$ ) in the tirofiban group. The median percent of residual stenosis prior to rescue treatment tended to be higher in the tirofiban group (80 [71-86] vs. 83 [79-90], $p=0.056$ ). Final reperfusion success (modified Treatment In Cerebral Ischemic 2b-3) was more frequent (42.4\%vs. 86.4\%, $p=$ $0.016)$ and post-procedure parenchymal hematoma type 2 and/or thick subarachnoid hemorrhages were less frequent $(15.3 \% \mathrm{vs} .5 .1 \%, p=0.068)$ in the tirofiban group. The frequency of favorable outcomes 3 months after endovascular treatment (modified Rankin Scale 0-2) was significantly higher in the tirofiban group (32.2\% vs. 52.5\%, $p=0.025$ ), and tirofiban administration was an independent predictor of favorable outcomes (odds ratio, $2.991 ; 95 \%$ confidence interval, 1.011-8.848; $p=0.048$ ).
\end{abstract}

Conclusions: Local tirofiban infusion can be a feasible adjuvant treatment option for patients with ICAS-LVO.

Keywords: Ischemic stroke, Intracranial atherosclerosis, Thrombectomy

\footnotetext{
*Correspondence: yangha.hwang@gmail.com; jinsoo22@gmail.com

'Department of Neurology, School of Medicine, Kyungpook National

University, 130 Dongdeok-ro, Jung-gu, Daegu 41944, South Korea

${ }^{7}$ Department of Neurology, Ajou University Medical Center, Ajou University

School of Medicine, 164 World cup-ro, Yeongtong-gu, Suwon 16499, South

Korea

Full list of author information is available at the end of the article
}

(c) The Author(s). 2020 Open Access This article is licensed under a Creative Commons Attribution 4.0 International License, which permits use, sharing, adaptation, distribution and reproduction in any medium or format, as long as you give appropriate credit to the original author(s) and the source, provide a link to the Creative Commons licence, and indicate if changes were made. The images or other third party material in this article are included in the article's Creative Commons. licence, unless indicated otherwise in a credit line to the material. If material is not included in the article's Creative Commons licence and your intended use is not permitted by statutory regulation or exceeds the permitted use, you will need to obtain permission directly from the copyright holder. To view a copy of this licence, visit http://creativecommons.org/licenses/by/4.0/ The Creative Commons Public Domain Dedication waiver (http://creativecommons.org/publicdomain/zero/1.0/) applies to the data made available in this article, unless otherwise stated in a credit line to the data. 


\section{Background}

Since randomized controlled trials for mechanical thrombectomy (MT) were successful, endovascular revascularization therapy (ERT) has been established as a standard treatment for acute ischemic stroke (AIS) with large vessel occlusion (LVO) of the intracranial anterior circulation [1-5]. MT is mostly based on stent retrieval or contact aspiration, which are designed for removing embolic clots in the occlusion vessel. However, if the occlusion is caused by intracranial atherosclerotic stenosis (ICAS), these MT methods may not be sufficient for recanalization and reperfusion, and rescue treatment is frequently required following MT [6-11]. Until now, angiographic and clinical outcomes of ERT for ICASLVO have been reported to be challenging [12-14].

ICAS is a common cause of stroke, especially in Asian populations $[15,16]$. In situ thrombosis (IST) is a major mechanism involved in emergent ICAS-LVO [17, 18]. In addition, the endothelium of the ICAS can be injured by MT [19, 20]. This thrombogenic milieu can cause thrombus propagation or reocclusion even after partial recanalization $[6,9,21,22]$. Therefore, stabilization of thrombogenic lesions should be considered for ICASrelated LVO.

In the current Tirofiban for Acute Serious Stroke Due to Intracranial in situ Thrombosis (Tirofiban ASSIST) study, we hypothesized that tirofiban, a locally infused antiplatelet agent, would stabilize the thrombogenic lesion in ICAS-LVO and improve clinical and angiographic outcomes. Therefore, we aimed to evaluate the safety and efficacy of intra-arterial tirofiban administration during ERT and to identify if this treatment is a predictor of favorable clinical outcomes in ICAS-LVO.

\section{Methods}

\section{Patients}

In this retrospective case-control study, the patients were recruited from the Acute Stroke due to Intracranial Atherosclerotic occlusion and Neurointervention Korean Retrospective (ASIAN KR) registry, which included databases from three stroke centers in Korea (from January 2011 to February 2016) [23]. Before data integration, all ASIAN KR data were de-identified. The criteria for inclusion were as follows: (1) patients had acute occlusion of the intracranial internal carotid artery (ICA), middle cerebral artery (MCA) M1, MCA M2, and vertebrobasilar artery; (2) the time from symptom onset to groin puncture was within 24h; and (3) patients were diagnosed with ICAS-LVO, which was retrospectively evaluated on the cerebral angiography as the etiology of stroke.

Patients were excluded if (1) the extracranial target arterial occlusion and/or tandem intracranial large arterial occlusion was present, (2) there were undetermined angiographic etiologies because the occlusion was never recanalized during primary MT, or (3) if patients had other etiologies of stroke, including vasculitis, arterial dissection, or Moyamoya disease.

The institutional review board in each center approved this study. The requirement for informed consent was waived because of the retrospective nature of this study. Intra-arterial tirofiban in Korea has been used for ERT with approval from the Korean Food and Drug Administration for each institution.

\section{Etiologic classification of target arterial lesion}

The etiology of target arterial lesion was classified based on angiographic findings during ERT. If there was no residual stenosis on angiography after reperfusion, the etiology was classified as an embolism $[9,22]$. In contrast, ICAS was defined using the following conditions: (1) presence of residual stenosis over $70 \%$ and (2) reocclusion tendency or flow impairment with residual stenosis less than $70 \%[9,22]$. If recanalization was not achieved throughout the ERT or without angioplasty or stenting, it was classified as intractable. The etiologic classification was performed by two experienced stroke neurologists, and a consensus was reached (Y.H.H. and J.S.L.).

\section{Endovascular procedures}

Stent retrieval and contact aspiration were mainly performed as primary MT strategies. If successful reperfusion was achieved but remnant ICAS was seen, followup angiography was performed 10-30 min after reperfusion. If the stenosis was aggravated, distal flow stagnation developed, or reocclusion occurred, repetitive MT or other rescue treatments, including switching MT strategy, intracranial tirofiban infusion, and balloon angioplasty and/or stenting, were applied. The decision of rescue treatment strategies was based on the neurointerventionists' discretion.

Patients in the tirofiban group were locally administered with $0.5 \mathrm{mg}$ to $2.0 \mathrm{mg}$ of tirofiban as a rescue treatment. Additionally, $0.5 \mathrm{mg}(2 \mathrm{ml})$ of tirofiban was diluted with $8 \mathrm{ml}$ of normal saline or $1 \mathrm{mg}(4 \mathrm{ml})$ of tirofiban with $6 \mathrm{ml}$ of normal saline for intra-arterial local infusion, and the $10 \mathrm{ml}$ of diluted tirofiban was manually administered approximately at a rate of $1 \mathrm{ml} / \mathrm{min}$ [6].

\section{Clinical and angiographic data}

We analyzed the clinical and demographic data of the patients, including National Institute of Health Stroke Scale (NIHSS) scores, Alberta Stroke Program Early CT Scores (ASPECTS), and pre-stroke modified Rankin Scale (mRS) scores at admission. The Arterial Occlusive Lesion (AOL) grade was used for the measurement of recanalization in the target arterial lesion, and AOL grade 2-3 was considered an indicator of successful recanalization [24]. The degree of remnant stenosis prior 
to rescue treatment was estimated by the WarfarinAspirin Symptomatic Intracranial Disease method [25]. Successful reperfusion was defined as a modified Treatment In Cerebral Ischemia (mTICI) score of $2 b$ or 3 based on the final angiography [24]. Brain CT was performed immediately and $12-24 \mathrm{~h}$ after ERT to evaluate hemorrhagic complications. Intracranial hemorrhages were classified based on the European Cooperative Acute Stroke Study [26]. Subarachnoid hemorrhage (SAH) severity was graded according to the modified Fisher scale [27]. Serious hemorrhagic complications were defined as parenchymal hematoma type 2 and/or a thick SAH with or without intraventricular hemorrhage (modified Fisher grade 3 or 4 of SAH). Postprocedural final infarct volume was measured by diffusion-weighted imaging (J.W.C.) using NordicICE semi-automated software (NordicNeuroLab, Bergen, Norway). Clinical outcomes were evaluated with mRS at 3 months after ERT. The mRS score was assessed by a certified neurologist or research nurse in each center during outpatient visit at 3 months after ERT. For patients who were unable to visit the outpatient department, structured telephone interview with the patient or family was conducted. A favorable clinical outcome was defined as an $\mathrm{mRS}$ score of $\leq 2$ or no change compared with the premorbid mRS.

\section{Statistics}

Chi-square tests or Fisher's exact tests were used for categorical variables. Mann-Whitney $U$ tests were used for continuous variables. A binary logistic regression analysis was performed to identify whether local tirofiban administration was an independent predictor of favorable clinical outcomes at 3 months and serious hemorrhagic complications. Age, sex, balloon angioplasty and/or stenting, and variables with $p<0.20$ in the univariate analysis were included in the binary logistic regression analysis for favorable clinical outcomes at 3 months. For serious hemorrhagic complications, onsetto-reperfusion time and variables with $p<0.20$ in the univariate analysis were included in the binary logistic regression analysis. For all analyses, $p<0.05$ was considered statistically significant. Statistical analyses were performed using SPSS 22.0 (IBM, Armonk, NY).

\section{Results}

Demographics and baseline characteristics

A total of 119 patients were included in this study (Fig. 1). Among them, 59 patients received local tirofiban infusion as a rescue treatment. Baseline characteristics and stroke risk factors are compared in Table 1. The median age of the patients was higher in the tirofiban group than in the non-tirofiban group (non-tirofiban group versus tirofiban group; 63 [55-75] versus 71 [61$78], p=0.015)$. The median initial NIHSS scores (15 [12-21] versus 14 [10-20], $p=0.322)$ and the median ASPECTS scores ( 8 [4.5-9.5] vs. 8 [6-9], $p=0.530)$ did not significantly differ between the two groups. Further, the use of intravenous recombinant tissue plasminogen activator (rtPA) did not significantly differ between the two groups $(49.2 \%$ versus $33.9 \%, p=0.093)$. Although

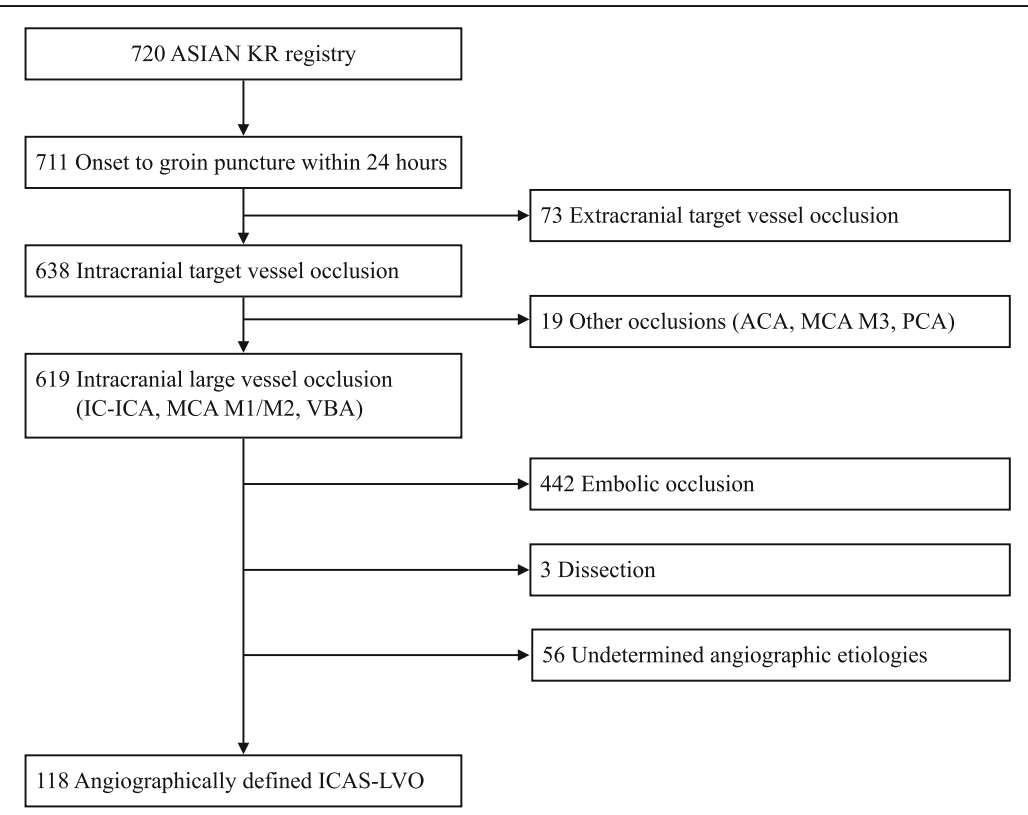

Fig. 1 Flowchart of this study. ACA, anterior cerebral artery; ASIAN KR, Acute Stroke due to Intracranial Atherosclerotic occlusion and Neurointervention Korean Retrospective; ICAS, intracranial atherosclerotic stenosis; IC-ICA, intracranial internal carotid artery; LVO, large vessel occlusion; MCA, middle cerebral artery; VBA, vertebrobasilar artery 
Table 1 Comparison of the baseline characteristics of the patients in the tirofiban and non-tirofiban groups

\begin{tabular}{|c|c|c|c|}
\hline & Non-tirofiban group $(n=59)$ & Tirofiban group $(n=59)$ & $P$ value \\
\hline Age, median (IQR) & $63(55-75)$ & $71(61-78)$ & 0.015 \\
\hline Female & $16(27.1 \%)$ & $23(39.0 \%)$ & 0.171 \\
\hline Prestroke mRS, median (IQR) & $0(0-0)$ & $0(0-0)$ & 0.438 \\
\hline Initial NIHSS, median (IQR) & $15(12-21)$ & $14(10-20)$ & 0.322 \\
\hline ASPECTS, median (IQR) & $8(4.5-9.5)(n=41)$ & $8(6-9)(n=46)$ & 0.530 \\
\hline Intravenous rtPA & $29(49.2 \%)$ & 30 (33.9\%) & 0.093 \\
\hline Target occlusion location & & & 0.766 \\
\hline Terminal ICA & $9(15.3 \%)$ & $8(13.6 \%)$ & \\
\hline MCA M1 & $34(57.6 \%)$ & $38(64.4 \%)$ & \\
\hline MCA M2 & $2(3.4 \%)$ & $3(5.1 \%)$ & \\
\hline VBA & $14(23.7 \%)$ & $10(16.9 \%)$ & \\
\hline Hypertension & $38(64.4 \%)$ & $38(64.4 \%)$ & $>0.999$ \\
\hline Diabetes mellitus & $19(32.2 \%)$ & $18(30.5 \%)$ & 0.843 \\
\hline Dyslipidemia & $25(42.4 \%)$ & $14(23.7 \%)$ & 0.031 \\
\hline Atrial fibrillation & $12(20.3 \%)$ & $9(15.3 \%)$ & 0.470 \\
\hline Coronary disease & $3(5.1 \%)$ & $4(6.8 \%)$ & $>0.999^{a}$ \\
\hline Smoking & $21(35.6 \%)$ & $23(39.0 \%)$ & 0.703 \\
\hline Prior antiplatelet & $6(10.2 \%)$ & $13(22.0 \%)$ & 0.080 \\
\hline Prior anticoagulant & $4(6.8 \%)$ & $1(1.7 \%)$ & $0.364^{\mathrm{a}}$ \\
\hline
\end{tabular}

ASPECTS Alberta Stroke Program Early CT Score, ICA internal carotid artery, IQR interquartile range, MCA middle cerebral artery, mRS modified Rankin Scale, NIHSS National Institute of Health Stroke Scale, rtPA recombinant tissue plasminogen activator, VBA vertebro-basilar artery

${ }^{\text {a } F i s h e r ' s ~ e x a c t ~ t-t e s t ~}$

the incidence of dyslipidemia was higher in the nontirofiban group than in the tirofiban group (42.4\% versus $23.7 \%, p=0.031$ ), other risk factors of stroke did not significantly differ between the two groups.

\section{Comparisons of angiographic data and outcomes}

The procedural, angiographic, and clinical outcomes for each group are summarized in Table 2. The median time from stroke symptom onset to groin puncture was shorter in the non-tirofiban group than in the tirofiban group (275 min versus $395 \mathrm{~min}, p=0.036$ ). The rate of aspiration thrombectomy and stent retriever thrombectomy, which were used as primary MT strategies, was similar in both groups. Compared to the non-tirofiban group, the median percent of remnant stenosis prior to rescue treatment tended to be higher in the tirofiban group (80 [71-86] versus 83 [79-90], $p=0.056$ ). Additional rescue treatments such as thrombectomy device switching, balloon angioplasty, and/or stenting were used more frequently in the non-tirofiban group than in the tirofiban group. However, no significant difference was found between the two groups with respect to the use of these treatments, with the exception of intracranial balloon angioplasty. No differences were noted in the rate of successful recanalization graded by AOL between the two groups $(69.5 \%$ versus $69.5 \%, p>0.999)$; however, the rate of successful reperfusion graded by mTICI was higher in the tirofiban group than in the non-tirofiban group $(42.4 \%$ versus $86.4 \%, p=0.016)$. Additionally, the incidence of SAH $(p=0.027)$ and intraventricular hemorrhage $(p=0.032)$ was higher in the non-tirofiban group than in the tirofiban group, but the occurrence of intracerebral hemorrhage did not differ between the groups $(p=0.311)$. The final infarct volume after ERT was smaller in the tirofiban group than in the non-tirofiban group ( $38.8 \mathrm{ml}$ versus $18.5 \mathrm{ml}, p=0.023$ ).

Repeat angiographies during admission after ERT were obtained in 32 patients in the non-tirofiban group and in 45 in the tirofiban group. The incidence of postprocedural reocclusion was significantly higher in the nontirofiban group than in the tirofiban group $(37.5 \%$ versus $4.4 \%, p<0.001)$. A favorable outcome 3 months after ERT was more frequent in the tirofiban group than in the non-tirofiban group ( $32.2 \%$ versus $52.5 \%, p=0.025)$.

Using a logistic regression model, local tirofiban infusion $(p=0.048)$ was found to be an independent predictor of favorable clinical outcomes (Table 3). In another regression model, local tirofiban infusion was not associated with serious hemorrhagic complications; however, the final infarct volume $(p=0.033)$ was independently associated with serious hemorrhagic complications (Table 4). Additionally, no significant interaction 
Table 2 Details of the endovascular treatment and clinical outcomes

\begin{tabular}{|c|c|c|c|}
\hline & Non-tirofiban group $(n=59)$ & Tirofiban group $(n=59)$ & $P$ value \\
\hline Onset-to-puncture time & $275(210-482)$ & $395(274-580)$ & 0.036 \\
\hline Puncture-to-final angiography time & $81(60-101)$ & $65(42-108)$ & 0.064 \\
\hline Onset-to-reperfusion time & $380(298-646)$ & $467(345-675)$ & 0.066 \\
\hline First-line endovascular treatment & & & 0.096 \\
\hline Aspiration thrombectomy & $29(49.2 \%)$ & $33(55.9 \%)$ & \\
\hline Stent retriever & $23(39.0 \%)$ & $25(42.4 \%)$ & \\
\hline Local fibrinolytics & $1(1.7 \%)$ & $1(1.7 \%)$ & \\
\hline Angioplasty & $6(10.2 \%)$ & 0 & \\
\hline Immediate reocclusion after first endovascular method & $10(17.9 \%)$ & $24(41.4 \%)$ & 0.006 \\
\hline Degree of residual stenosis prior to rescue treatment (\%) & $80(71-86)$ & $83(79-90)$ & 0.056 \\
\hline \multicolumn{4}{|l|}{ Rescue treatments } \\
\hline Local tirofiban infusion only & 0 & $48(81.4 \%)$ & $<0.001$ \\
\hline Stent retriever to aspiration & $1(1.7 \%)$ & 0 & $>0.999^{a}$ \\
\hline Aspiration to stent retriever & $8(13.6 \%)$ & $3(5.1 \%)$ & 0.113 \\
\hline Intracranial balloon angioplasty & $9(15.3 \%)$ & $2(3.4 \%)$ & 0.027 \\
\hline Intracranial stenting & $12(20.3 \%)$ & $6(10.2 \%)$ & 0.124 \\
\hline Final AOL 2-3 & $41(69.5 \%)$ & $41(69.5 \%)$ & $>0.999$ \\
\hline Final mTICl 2b-3 & $25(42.4 \%)$ & $51(86.4 \%)$ & 0.016 \\
\hline Postprocedural reocclusion & $12(37.5 \%, n=32)$ & $2(4.4 \%, n=45)$ & $<0.001$ \\
\hline Intracerebral hemorrhage & & & 0.311 \\
\hline HT type 1 & $4(6.8 \%)$ & $2(3.4 \%)$ & \\
\hline HT type 2 & $6(10.2 \%)$ & $3(5.1 \%)$ & \\
\hline PH type 1 & $3(5.1 \%)$ & $1(1.7 \%)$ & \\
\hline PH type 2 & $6(10.2 \%)$ & $3(5.1 \%)$ & \\
\hline Subarachnoid hemorrhage & $6(10.2 \%)$ & 0 & $0.027^{\mathrm{a}}$ \\
\hline Intraventricular hemorrhage & $8(13.6 \%)$ & $1(1.7 \%)$ & $0.032^{\mathrm{a}}$ \\
\hline Serious hemorrhagic complication ${ }^{b}$ & $9(15.3 \%)$ & $3(5.1 \%)$ & 0.068 \\
\hline Final infarct volume, $\mathrm{ml}$ (median, IQR) & $38.8(14.3-92.7)$ & $18.5(7.9-37.2)$ & 0.023 \\
\hline mRS $0-2$ at 3 months & 19 (32.2\%) & 31 (52.5\%) & 0.025 \\
\hline Mortality & $12(20.3 \%)$ & $4(6.8 \%)$ & 0.031 \\
\hline
\end{tabular}

$\overline{A O L}$ arterial occlusive lesion, ERT endovascular revascularization therapy, $H T$ hemorrhagic transformation, $m R S$ modified Rankin Scale, $M T$ mechanical thrombectomy, $m T / C l$ modified treatment in cerebral ischemia, $P H$ parenchymal hematoma

${ }^{\mathrm{a}}$ Fisher's exact t-test; ${ }^{\mathrm{b}}$ Serious hemorrhagic complications consist of parenchymal hematoma type 2 and/or subarachnoid hemorrhage Fisher grade 3-4

was found between tirofiban infusion and final infarct volume for serious hemorrhagic complications $(p=$ $0.339)$.

\section{Discussion}

In this study, we evaluated the safety and efficacy of local tirofiban infusion as a rescue ERT strategy for AIS for patients with ICAS-LVO. The main findings of this study were as follows: (1) the rates of successful reperfusion and favorable outcomes were higher in the tirofiban group than in the non-tirofiban group, and (2) despite its lytic characteristics, whereas the rate of hemorrhagic complications appeared to be the result of the final large infarct volume, it was lower in the tirofiban group than in the non-tirofiban group. Overall, results from this retrospective registry study suggested that local tirofiban infusion could be a safe and effective rescue treatment for patients with ICAS-LVO.

ICAS is a major etiology of LVO, especially in Asian populations, and is still challenging to manage during modern MT [12, 17, 22]. ICAS-related LVO may result from IST beyond a preexisting stenosis [6, 21, 22, 28]. In IST, the rupture of preexisting atherosclerotic plaques and the release of tissue factors from the endothelial surface can lead to a thrombogenic and platelet aggravating environment [18]. In addition, usual MT may induce 
Table 3 Binary logistic regression analysis for favorable clinical outcomes

\begin{tabular}{lll}
\hline Variables & Odds ratio (95\% Cl) & $p$ value \\
\hline Age & $0.920(0.871-0.971)$ & 0.002 \\
Female & $0.616(0.205-1.849)$ & 0.388 \\
Baseline NIHSS & $0.851(0.772-0.939)$ & 0.001 \\
Occlusion location & & 0.269 \\
$\quad$ Terminal ICA & Ref. & 0.064 \\
MCA M1 & $5.109(0.911-28.663)$ & 0.268 \\
MCA M2 & $3.397(0.239-48.368)$ & 0.310 \\
$\quad$ VBA & $2.775(0.387-19.921)$ & 0.818 \\
Onset to puncture time & $1.000(0.999-1.001)$ & 0.112 \\
Puncture to final reperfusion time & $0.990(0.978-1.002)$ & 0.261 \\
Successful reperfusion & $1.986(0.552-7.149)$ & 0.079 \\
Rescue balloon angioplasty and/or stenting & $0.288(0.072-1.157)$ & 0.048 \\
Local tirofiban infusion & $2.991(1.011-8.848)$ &
\end{tabular}

ICA internal carotid artery, MCA middle cerebral artery, NIHSS National Institute of Health Stroke Scale, VBA vertebro-basilar artery

plaque rupture and cause extensive arterial injury from the endothelium to the tunica media $[19,20]$. Therefore, local thrombogenic conditions may be exacerbated, and this often causes the vessel to become reoccluded even after successful reperfusion is achieved by usual MT. Based on these data, early stabilization of the endothelium and intracranial atherosclerotic plaque is an important goal, and antiplatelet administration is ideal to stabilize the thrombogenic lesion. Since the underlying ICAS is hidden in LVO, pretreatment with oral antiplatelet agents cannot be applied in most cases; thus, infusible antiplatelet has been anecdotally used in the IST lesion as rescue treatment for intracranial LVO [6, $29,30]$. To this end, the glycoprotein IIb/IIIa inhibitor may play a crucial role in the prevention of fibrinogeninduced platelet aggregation and local thrombus formation [31].

Tirofiban is an infusible antiplatelet glycoprotein IIb/ IIIa inhibitor. It has been indicated for unstable angina and myocardial infarction [31]. Compared with another glycoprotein IIb/IIIa inhibitor, abciximab, which is an irreversible antiplatelet, tirofiban is a reversible antiplatelet [31]. Given the relatively long platelet recovery time of abciximab (up to $48 \mathrm{~h}$ ), hemorrhagic complications are of greater concern for abciximab than for tirofiban (up to 2-4h) [32]. While another glycoprotein IIB/IIIA inhibitor, eptifibatide, is not available in Korea, the use of tirofiban in ERT has been approved by the Korean Food and Drug Administration for emergency setting.

In the current study, we evaluated revascularization status using AOL and mTICI scale which could assess different dimensions such as recanalization and reperfusion, respectively. The AOL scale can assess directly the performance of MT and can be useful for the estimation of the remnant stenosis because the AOL scale measures the recanalization status at the target occlusive lesion (none, incomplete, complete) [24]. However, it may be possible to ignore the status of the target downstream territory. On the contrary, the mTICI scale estimates the antegrade restoration of the capillary blush so that it could estimate the extent of reperfusion of the target downstream territory [24] and could be advantageous to reflect the thrombogenic events such as thrombus propagation and distal embolization by IST. In this study, we found an important role of tirofiban in reperfusion beyond recanalization. In terms of recanalization,

Table 4 Binary logistic regression analysis for serious hemorrhagic complications

\begin{tabular}{lll}
\hline Variables & Odds ratio $(95 \% \mathrm{Cl})$ & $p$ value \\
\hline Age & $0.965(0.894-1.042)$ & 0.361 \\
Intravenous rtPA & $0.228(0.019-2.804)$ & 0.248 \\
Prior use of oral antiplatelet or anticoagulant & $1.624(0.133-19.880)$ & 0.705 \\
Onset to final reperfusion time & $0.999(0.994-1.003)$ & 0.620 \\
Local tirofiban & $1.362(0.123-15.102)$ & 0.801 \\
Final infarct volume & $1.010(1.001-1.019)$ & 0.033 \\
\hline
\end{tabular}

ERT endovascular revascularization therapy, rtPA recombinant tissue plasminogen activator 
the rate of successful recanalization graded by AOL was the same in both groups (69.5\%, respectively). However, the ERT procedure was completed in $81.4 \%$ of the patients only after tirofiban was locally injected as a single rescue treatment in the tirofiban group. In addition, the incidence of postprocedural reocclusion on repeat angiographies was much lower in the tirofiban group than in the non-tirofiban group even though the degree of remnant stenosis prior to rescue treatment tended to be higher in the tirofiban group. These findings suggest that tirofiban may stabilize the thrombogenic environment in the stenotic lesion and reduce the use of additional MT strategies. Subsequently, endothelial damage and endovascular procedure time may also be reduced.

Beyond recanalization, the reperfusion status should always be considered. Reperfusion includes restoration of blood flow into the distal branches and the deep brain $[24,33]$. In this study, even if both groups had the same rate of successful recanalization, the rate of successful reperfusion was higher in the tirofiban group than in the non-tirofiban group. In most cases, tirofiban infusion was administered immediately after the first partial recanalization in cases with a suspicion of underlying stenosis or in cases of reocclusion after recanalization. Early local tirofiban infusion may contribute to the prevention of downstream embolization by local thrombosis, which may result in a better reperfusion status [34].

Multiple studies have reported that the application of glycoprotein IIb/IIIa inhibitors increases the risk of postprocedural hemorrhagic complications. Although glycoprotein IIb/IIIa inhibitors are not fibrinolytic agents, a high rate of fatal intracerebral hemorrhages has been reported $[35,36]$. These studies reported that glycoprotein IIb/IIIa inhibitors were administered intravenously for at least $12 \mathrm{~h}$. A relatively high dose of glycoprotein IIb/IIIa inhibitors may be needed to elicit the appropriate action when it is administered intravenously. In addition, because patients were enrolled up to 2011 in these studies, new MT techniques may have not been incorporated. Further, similar to the failed ERT trials in 2013 [37-39], the rate of successful reperfusions in these studies was relatively low (61.6\% in the tirofiban study). Lower rates of successful reperfusion may be related to a greater final infarct volume, which may be more vulnerable to antithrombotic therapy. In contrast, the present results revealed that tirofiban did not increase intracerebral hemorrhages when it was slowly infused via catheter and administered at a low dose following newer MT treatment. Recent studies have demonstrated that primary stent retrieval effectively removed in situ thrombi in ICAS-LVO [8, 40]. On the other hand, serious hemorrhagic complications were more strongly associated with the final infarction volume than with intravenous thrombolysis or local tirofiban infusion shown in the present study. Our results suggest that the appropriate administration of tirofiban may maintain the reperfusion status and reduce the infarct volume. Therefore, the risk of serious hemorrhagic complications may be reduced following tirofiban administration.

This study had several limitations. First, given the retrospective design with a relatively small sample size, data may be skewed, and hidden confounders may have affected the direction of treatment. Additionally, a previous study reported that stenosis length affected treatment outcomes [41]. However, stenosis length could not be measured in the present study because of the interference caused by IST and LVO or vessel injury by primary MT. Nevertheless, our main results are supported by multivariable adjustments, which consisted of well-known predictors. Second, the dose and infusion speed of tirofiban was not prespecified because of the retrospective nature of this study. However, from early experiences and previous anecdotal reports, the dose did not vary extensively. For example, the total amount of tirofiban infusion was low and only varied from $0.5 \mathrm{mg}$ to $2.0 \mathrm{mg}$ among all three stroke centers. Additionally, the infusion speed was between 0.05 and $0.1 \mathrm{mg} / \mathrm{min}$. Third, although patients with LVO and underlying ICAS were included in this study, some patients also had atrial fibrillation whereas the frequency did not differ between groups. These cases may have contaminated the effectiveness and outcomes of local tirofiban infusion on IST of ICAS-LVO. To overcome this limitation, we conducted further analyses that excluded patients with atrial fibrillation (shown in the Supplemental document); however, the clinical and angiographic outcomes did not differ. Finally, old-generation contact aspiration catheters were used in some portions of the primary MT devices. However, the frequency of the use did not differ between the groups in our post-hoc analysis. Considering that the main goal of this study was to identify rescue ERT strategies for underlying ICAS after thrombectomy, the effect of MT devices would be minor.

\section{Conclusions}

Local tirofiban infusion following MT may be a feasible treatment option for patients with ICAS-LVO. The rate of favorable outcomes was higher and the rate of serious hemorrhagic complications was lower in patients who received tirofiban infusion as a rescue treatment than in patients who did not receive infusions of tirofiban as a rescue treatment.

\section{Supplementary information}

Supplementary information accompanies this paper at https://doi.org/10. 1186/s12883-020-01864-4.

Additional file 1: Table S1. Comparison of baseline characteristics between the tirofiban and non-tirofiban groups. Table S2. Details of 
endovascular treatment and clinical outcomes. Table S3. Binary logistic regression analysis for favorable clinical outcome. Table S4. Binary logistic regression analysis for serious hemorrhagic complications.

\section{Abbreviations}

AIS: Acute ischemic stroke; AOL: Arterial occlusive lesion; ASIAN KR: Acute Stroke due to Intracranial Atherosclerotic occlusion and Neurointervention Korean Retrospective; ASPECTS: Alberta Stroke Program Early CT Scores: ERT: Endovascular revascularization therapy; ICA: Internal carotid artery; ICAS: Intracranial atherosclerotic stenosis; IST: In situ thrombosis; LVO: Large vessel occlusion; MCA: Middle cerebral artery; mRS: Modified Rankin Scale; MT: Mechanical thrombectomy; mTICl: Modified Treatment In Cerebral Ischemia; NIHSS: National Institute of Health Stroke Scale; rtPA: Recombinant tissue plasminogen activator; SAH: Subarachnoid hemorrhage; Tirofiban ASSI ST: Tirofiban for Acute Serious Stroke Due to Intracranial in situ Thrombosis

\section{Acknowledgements}

Not Applicable.

\section{Authors' contributions}

Study design YWK, YHH, JSL; data collection YWK, SIS, JY, JHH, CHK, DHK, YSK, SJL, JMH, JWC, YHH, JSL; data analysis YWK, JSL; data interpretation YWK, YHH, JSL; preparation of the manuscript YWK, YHH, JSL; review and editing YWK, SIS, JY, JHH, CHK, DHK, YSK, SJL, JMH, JWC, YHH, JSL. All authors have read and approved the final version of manuscript.

\section{Funding}

The current study was supported by a grant from ASPEN GLOBAL INCORPORATED (J.S.L.); however, the funder had no role in the study design, data collection and analysis, decision to publish, or preparation of the manuscript.

\section{Availability of data and materials}

The datasets used and/or analyzed during the present study are available from the corresponding author on reasonable request.

\section{Ethics approval and consent to participate}

The protocol for data collection was approved by the Institutional Review Board (IRB) of each hospital (Ajou University Hospital IRB: AJIRB-MED-OBS-15483, AJIRB-MED-OBS-17-094: Keimyung University Dongsan Hospital IRB: 2016-01-038-009; Kyungpook National University Hospital IRB: 2016-01-020006). Our study was implemented in accordance with the ethical standards of the 1964 Declaration of Helsinki and its later amendments. The need for written informed consent was waived because of the retrospective nature of this study.

\section{Consent for publication}

Not Applicable.

\section{Competing interests}

The authors declare that they have no competing interests.

\footnotetext{
Author details

'Department of Neurology, School of Medicine, Kyungpook National University, 130 Dongdeok-ro, Jung-gu, Daegu 41944, South Korea. ${ }^{2}$ Department of Neurology, Keimyung University School of Medicine, Daegu, South Korea. ${ }^{3}$ Department of Neurology, National Health Insurance Service Ilsan Hospital, Goyang, South Korea. ${ }^{4}$ Department of Neurosurgery, Keimyung University School of Medicine, Daegu, South Korea. ${ }^{5}$ Department of Neurosurgery, School of Medicine, Kyungpook National University, Daegu, South Korea. ${ }^{6}$ Department of Radiology, School of Medicine, Kyungpook National University, Daegu, South Korea. ${ }^{7}$ Department of Neurology, Ajou University Medical Center, Ajou University School of Medicine, 164 World cup-ro, Yeongtong-gu, Suwon 16499, South Korea. ${ }^{8}$ Department of Radiology, Ajou University School of Medicine, Suwon, South Korea.
}

Received: 9 April 2020 Accepted: 14 July 2020

Published online: 20 July 2020

\section{References}

1. Berkhemer OA, Fransen PSS, Beumer D, van den Berg LA, Lingsma HF, Yoo $A J$, et al. MR CLEAN Investigators A randomized trial of intraarterial treatment for acute ischemic stroke. N Engl J Med. 2015;372:11-20.

2. Campbell BCV, Mitchell PJ, Kleinig TJ, Dewey HM, Churilov L, Yassi N, et al. EXTEND-IA investigators. Endovascular therapy for ischemic stroke with perfusion-imaging selection. N Engl J Med. 2015;372:1009-18.

3. Goyal M, Demchuk AM, Menon BK, Eesa M, Rempel JL, Thornton J, et al. ESCAPE Trial Investigators Randomized assessment of rapid endovascular treatment of ischemic stroke. N Engl J Med. 2015;372:1019-30.

4. Saver JL. Goyal M, Bonafé a, Diener H-C, levy El, Pereira VM, et al; SWIFT PRIME investigators. Stent-retriever thrombectomy after intravenous t-PA vs. t-PA alone in stroke. N Engl J Med. 2015;372:2285-95.

5. Jovin TG, Chamorro A, Cobo E, de Miquel MA, Molina CA, Rovira A, et al. REVASCAT trial investigators. Thrombectomy within 8 hours after symptom onset in ischemic stroke. N Engl J Med. 2015;372:2296-306.

6. Kang DH, Kim YW, Hwang YH, Park SP, Kim YS, Baik SK. Instant reocclusion following mechanical thrombectomy of in situ thromboocclusion and the role of low-dose intra-arterial tirofiban. Cerebrovasc Dis. 2014;37:350-5.

7. Yoon W, Kim SK, Park MS, Kim BC, Kang HK. Endovascular treatment and the outcomes of atherosclerotic intracranial stenosis in patients with hyperacute stroke. Neurosurgery. 2015;76:680-6.

8. Lee JS, Hong JM, Lee KS, Suh HI, Choi JW, Kim SY. Primary stent retrieval for acute intracranial large artery occlusion due to atherosclerotic disease. J Stroke. 2016;18:96-101.

9. Kim YW, Hong JM, Park DG, Choi JW, Kang DH, Kim YS, et al. Effect of intracranial atherosclerotic disease on endovascular treatment for patients with acute Vertebrobasilar occlusion. AJNR Am J Neuroradiol. 2016;37:20728.

10. Al Kasab S, Almadidy Z, Spiotta AM, et al. Endovascular treatment for AlS with underlying ICAD. J Neurointerv Surg. 2017;9:948-51.

11. Kang DH, Yoon W, Kim SK, et al. Endovascular treatment for emergent large vessel occlusion due to severe intracranial atherosclerotic stenosis. Neurosurg. 2019;130:1949-56.

12. Matias-Guiu JA, Serna-Candel C, Matias-Guiu J. Stroke etiology determines effectiveness of retrievable stents. J Neurointerv Surg. 2014;6:e11.

13. Lee JS, Lee S-J, Yoo JS, Hong J-H, Kim C-H, Kim Y-W, et al. Prognosis of acute intracranial atherosclerosis-related occlusion after endovascular treatment. J Stroke. 2018;20:394-403.

14. Tsang ACO, Orru E, Klostranec JM, Yang I-H, Lau KK, Tsang FCP, et al. Thrombectomy outcomes of intracranial atherosclerosis-related occlusions. Stroke. 2019;50:1460-6.

15. Wong LKS. Global burden of intracranial atherosclerosis. Int J Stroke. 2006;1: 158-9.

16. Bang OY. Intracranial atherosclerosis: current understanding and perspectives. J Stroke. 2014;16:27-35.

17. Kim JS, Nah H-W, Park SM, Kim S-K, Cho KH, Lee J, et al. Risk factors and stroke mechanisms in atherosclerotic stroke: intracranial compared with extracranial and anterior compared with posterior circulation disease. Stroke. 2012;43:3313-8.

18. Wong KSL, Caplan RL, Kim JS. Stroke mechanisms. In: Kim JS, Caplan LR, Wong KSL, editors. Intracranial atherosclerosis. Oxford: Wiley- Blackwell; 2008. p. 57-68.

19. Teng D, Pannell JS, Rennert RC, Li J, Li Y-S, Wong WW, et al. Endothelial trauma from mechanical thrombectomy in acute stroke: in vitro live-cell platform with animal validation. Stroke. 2015;46:1099-106.

20. Peschillo S, Diana F, Berge J, Missori P. A comparison of acute vascular damage caused by ADAPT versus a stent retriever device after thrombectomy in acute ischemic stroke: a histological and ultrastructural study in an animal model. J Neurointerv Surg. 2017:9:743-9.

21. Hwang YH, Kim YW, Kang DH, Kim YS, Liebeskind DS. Impact of target arterial residual stenosis on outcome after endovascular revascularization. Stroke. 2016;47:1850-7

22. Lee JS, Hong JM, Kim JS. Diagnostic and therapeutic strategies for acute intracranial atherosclerosis-related occlusions. J Stroke. 2017;19:143-51.

23. Lee JS, Lee S-J, Hong JM, Choi JW, Hong J-H, Chang H-W, et al. Temporal changes in care processes and outcomes for endovascular treatment of 
acute ischemic stroke: retrospective registry data from three Korean centers. Neurointervention. 2018;13:2-12.

24. Zaidat OO, Yoo AJ, Khatri P, Tomsick TA, von Kummer R, Saver JL, et al. Recommendations on angiographic revascularization grading standards for acute ischemic stroke. Stroke. 2013;44:2650-63.

25. Samuels OB, Joseph GJ, Lynn MJ, Smith HA, Chimowitz MI. A standardized method for measuring intracranial arterial stenosis. AJNR Am J Neuroradiol. 2000;21:643-6.

26. Fiorelli M, Bastianello S, von Kummer R, del Zoppo GJ, Larrue V, Lesaffre E, et al. Hemorrhagic transformation within 36 hours of a cerebral infarct: relationships with early clinical deterioration and 3-month outcome in the European cooperative acute stroke study I (ECASS I) cohort. Stroke. 1999;30: $2280-4$.

27. Frontera JA, Claassen J, Schmidt JM, Wartenberg KE, Temes R, Connolly ES, et al. Prediction of symptomatic vasospasm after subarachnoid hemorrhage: the modified fisher scale. Neurosurgery. 2006;59:21-7.

28. Chen Y-C, Huang AL, Kyaw TS, Bobik A, Peter K. Atherosclerotic plaque rupture: identifying the straw that breaks the Camel's Back. Arterioscler Thromb Vasc Biol. 2016;36:e63-72.

29. Zhao W, Che R, Shang S, Wu C, Li C, Wu L, et al. Low-dose Tirofiban improves functional outcome in acute ischemic stroke patients treated with endovascular Thrombectomy. Stroke. 2017:48:3289-94.

30. Yu T, Lin Y, Jin A, Zhang P, Zhou X, Fang M, et al. Safety and efficiency of low dose intra-arterial Tirofiban in mechanical Thrombectomy during acute ischemic stroke. Curr Neurovasc Res. 2018;15:145-50.

31. King S, Short M, Harmon C. Glycoprotein Ilb/llla inhibitors: the resurgence of tirofiban. Vasc Pharmacol. 2016;78:10-6.

32. Brown DL, Fann CS, Chang CJ. Meta-analysis of effectiveness and safety of abciximab versus eptifibatide or tirofiban in percutaneous coronary intervention. Am J Cardiol. 2001;87:537-41.

33. Cho T-H, Nighoghossian N, Mikkelsen IK, Derex L, Hermier M, Pedraza S, et al. Reperfusion within 6 hours outperforms recanalization in predicting penumbra salvage, lesion growth, final infarct, and clinical outcome. Stroke. 2015;46:1582-9.

34. Siebler M, Hennerici MG, Schneider D, von Reutern GM, Seitz RJ, Röther J, et al. Safety of Tirofiban in acute ischemic stroke: the SaTIS trial. Stroke. 2011:42:2388-92.

35. Adams HP, Effron MB, Torner J, Dávalos A, Frayne J, Teal P, et al. AbESTT-II Investigators. Emergency administration of abciximab for treatment of patients with acute ischemic stroke: results of an international phase III trial: Abciximab in Emergency Treatment of Stroke Trial (AbESTT-II). Stroke. 2008; 39:87-99.

36. Kellert L, Hametner C, Rohde S, Bendszus M, Hacke W, Ringleb P, et al. Endovascular stroke therapy: tirofiban is associated with risk of fatal intracerebral hemorrhage and poor outcome. Stroke. 2013;44:1453-5.

37. Broderick JP, Palesch YY, Demchuk AM, Yeatts SD, Khatri P, Hill MD, Interventional Management of Stroke (IMS) III investigators, et al. Endovascular therapy after intravenous t-PA versus t-PA alone for stroke. N Engl J Med. 2013;368:893-903.

38. Ciccone A, Valvassori L, Nichelatti M, Sgoifo A, Ponzio M, Sterzi R, et al. Endovascular treatment for acute ischemic stroke. N Engl J Med. 2013;368: 904-13.

39. Kidwell CS, Jahan R, Gornbein J, Alger JR, Nenov V, Ajani Z, MR RESCUE investigators, et al. A trial of imaging selection and endovascular treatment for ischemic stroke. N Engl J Med. 2013;368:914-23.

40. Lee JS, Lee S-J, Hong JM, Choi JW, Yoo J, Hong J-H, et al. Solitaire Thrombectomy for acute stroke due to intracranial atherosclerosis-related occlusion: ROSE ASSIST study. Front Neurol. 2018;9:1064.

41. Mori T, Fukuoka M, Kazita K, Mori K. Follow-up study after intracranial percutaneous transluminal cerebral balloon angioplasty. AJNR Am J Neuroradiol. 1998:19:1525-33.

\section{Publisher's Note}

Springer Nature remains neutral with regard to jurisdictional claims in published maps and institutional affiliations.

Ready to submit your research? Choose BMC and benefit from:

- fast, convenient online submission

- thorough peer review by experienced researchers in your field

- rapid publication on acceptance

- support for research data, including large and complex data types

- gold Open Access which fosters wider collaboration and increased citations

- maximum visibility for your research: over $100 \mathrm{M}$ website views per year

At $\mathrm{BMC}$, research is always in progress.

Learn more biomedcentral.com/submissions 